\title{
Completely model-free determination of the level density, radiative strength functions and their main peculiarities
}

\author{
A. Sukhovoj ${ }^{1, a}$, V. Khitrov ${ }^{1}$, and V. Maslov²,b \\ 1 Joint Institute for Nuclear Reaserch, 141980 Dubna, Russia \\ 2 Joint Institute for Nuclear and Energy Reaserch, 220109 Minsk-Sosny, Belarus
}

\begin{abstract}
The level density and radiative strength functions obtained for the compound nuclei ${ }^{40} \mathrm{~K},{ }^{60} \mathrm{Co},{ }^{71,74} \mathrm{Ge}$, ${ }^{80} \mathrm{Br},{ }^{114} \mathrm{Cd},{ }^{118} \mathrm{Sn},{ }^{124,125} \mathrm{Te},{ }^{128} \mathrm{I},{ }^{137,138,139} \mathrm{Ba},{ }^{140} \mathrm{La},{ }^{150} \mathrm{Sm},{ }^{156,158} \mathrm{Gd},{ }^{160} \mathrm{~Tb},{ }^{163,164,165} \mathrm{Dy},{ }^{166} \mathrm{Ho},{ }^{168} \mathrm{Er},{ }^{170} \mathrm{Tm},{ }^{174} \mathrm{Yb}$, ${ }^{176,177} \mathrm{Lu},{ }^{181} \mathrm{Hf},{ }^{182} \mathrm{Ta},{ }^{183,184,185,187} \mathrm{~W},{ }^{188,190,191,193} \mathrm{Os},{ }^{192} \mathrm{Ir},{ }^{196} \mathrm{Pt},{ }^{198} \mathrm{Au},{ }^{200} \mathrm{Hg}$ precisely reproduce the intensities of two-step cascades, density and total radiation widths of neutron resonance. For 21 nuclei cascade populating ability $\sim 100$ of their excited levels were also reproduced. The averaged strength functions values and the deviations of the level density from the interpolating exponential dependence suggest that below $B_{n}$ a sharp change in the structure of excited levels is observed at two excitation energies at least.
\end{abstract}

\section{Introduction}

Presently, the information on level densities $\rho$ has been mainly obtained in the framework of Bohr-Mottelson hypothesis [1] on independence of partial cross section of interaction of reaction product on a residual nucleus with excitation energy $U$. In the one-step reactions (spectrum of evaporative nucleons, for example) at the determination of $\rho$ the systematic error directly depends on the degree of its inaccuracy.

Partial cross sections of two-step reactions, such as nucleon or gamma-quantum, gamma-quantum onto low lying level, weakly depend on that hypothesis. It is caused by the following circumstances:

a) the partial gamma widths $\Gamma_{\lambda i}$ for the gamma-quanta are proportional to $E_{\gamma}^{3}$;

b) total radiative width $\Gamma_{i}$ of the levels, excited at the first step, is the sum of the partial gamma widths. Therefore the deviation of values of the different signs from the general trend is effectively reduced in the sum.

Moreover, two-step reactions make it possible to estimate a degree of deviation of the interaction cross section $\sigma(E, U \neq 0)$ of final nucleus from its value $\sigma(E, U=0)$.

It is just the measurement of cascades intensity $i_{\gamma \gamma}$ from two gamma-quanta at coincidences through a nucleus individual levels together with the data acquisition on total intensity of primary $i_{1}$ and secondary $i_{2}$ cascade gammaquanta that permitted [2] to firstly reveal and estimate the degree of dependence $\sigma(E, U)$ to be lower than $\approx 0.5 B_{n}$. The congruent analysis showed that a very strong and specific degree of energy dependence of the interaction cross section of gamma-quantum with the residual nucleus appears for all studied nuclei at their excitation energies $U>2-5 \mathrm{MeV}$.

a Presenting author, e-mail: suchovoj@nf.jinr.ru

${ }^{b}$ Presenting author, e-mail: maslov@bas-net.by

\section{Main principles of analysis}

The registration of the two-step cascades at the thermal neutrons capture makes possible to experimentally [3] determine their intensity for all possible energies of primary gamma-transitions $E_{1}$ with an insignificant [4] inaccuracy form of the dependence of cascades intensity on energy of their primary gamma-transition. Probably, its total systematical error does not exceed $20-30 \%$ in the majority of studied nuclei. Coefficients of this error transfer have different character [5] at various excitation energies. Their maximum value does not exceed $5-10$ by module and they are very small in the region of half excitation energy of the studied nucleus.

It is due to the cascades registration only in peaks of full energy capture by a pair of HPGe-detectors and a strong changing in number of obtained cascades and their intensity, when there is a change of the primary gamma-transition energy.

For the fixed energies of primary and final cascade levels and arbitrary energy interval $\Delta E_{i}$ with $n_{i}$ intermediate levels the summed cascade intensity is determined by equation:

$$
i_{\gamma \gamma}\left(E_{1}\right)=\frac{\Gamma_{\lambda i}}{\Gamma_{\lambda}} n_{i} \frac{\Gamma_{i f}}{\Gamma_{i}} .
$$

It may be additionally summed by various initial $\lambda$ and final $f$ levels of cascades.

A complex of information on:

- intensity of two-step cascades $I_{\gamma \gamma}=\sum_{\lambda f} i_{\gamma \gamma}$;

- total radiative width $\Gamma_{\lambda}$ of their initial level;

- cascade populating ability $P=i_{1} i_{2} / i_{\gamma \gamma}$ of several tens (hundreds and more) of individual levels $i$;

- density of neutron resonances and known number $n_{f}$ of low lying levels

may be reproduced (without using the hypothesis [1]) by an infinite number of different random functions $\rho$ and $k=\left\langle\Gamma_{\lambda i}\right\rangle /\left(E_{\gamma}^{3} A^{2 / 3} D_{\lambda}\right)$ with similar and very small $\left(\chi^{2} / f \ll 1\right)$ values for criterion of the approximation quality. System of 

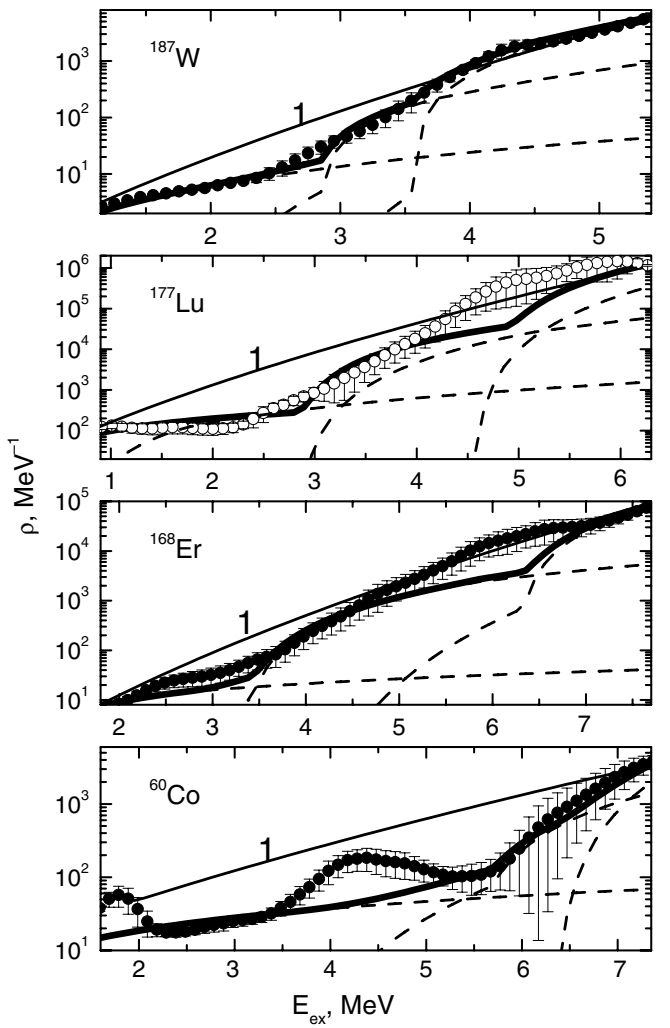

Fig. 1. Line 1 corresponds to predictions of the model described in [6]. The circles with error bars are the level densities, which reproduce the set of experimental data as a function of the energy of cascade intermediate level. Dashed lines show best fit of partial level densities of 2, 4, 6 quasiparticle excitations (even-A) and 3, 5, 7 (odd-A). Thick line - sum of the partial level densities. Full and open points - data obtained without the use and with the use of a hypothesis [1].

functional equations (1) describes a closed surface of small volume in the space of $2 \mathrm{~N}$ unknown parameters. In that case there are always minimum and maximum possible values of parameters $\rho$ and $k$, which may reproduce a whole all set of the listed above experimental data. Due to the nonlinearity of system (1) and strong correlations of its parameters, the ratios of maximum and minimum possible values of $\rho$ and $k$ are strongly limited for nucleus excitation and product of two-step reaction energies.

Any number of the random functions $\rho$ and $k$ from this region may be simultaneously determined from the experimental data by Monte Carlo method. For example, parameters compromising between speed of reaching the minimum $\chi^{2}$ and refine reproduction shape of functions $\rho$ and $k$ are presented in [6].

\section{General characters of the experimental data for $\rho$ and $k$}

The sections ratio $\sigma(E, U) / \sigma(E, U=0)$ is less than one in the region of $U \sim 1-3 \mathrm{MeV}$ and it is more - about $0.5 B_{n}$ for the majority of studied in [2] nuclei. This circumstance makes it

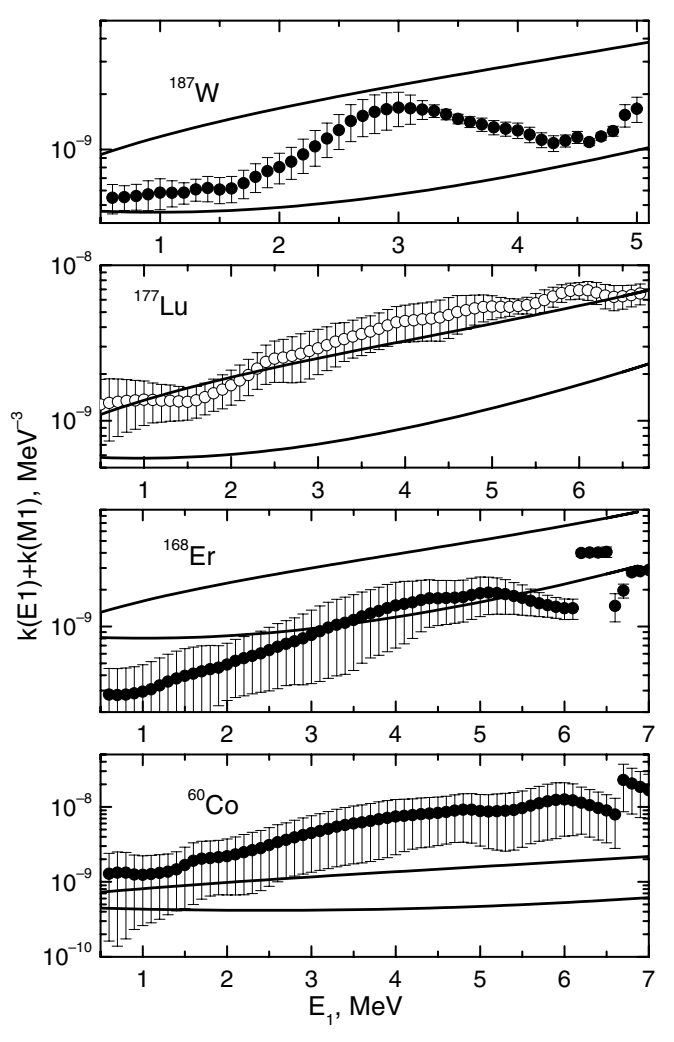

Fig. 2. The circles with error bars are the sums of the radiative strength functions of dipole electric and magnetic transitions of cascades, which allow us to accurately reproduce the above listed experimental data. Upper line corresponds the model, described in [7], lower line is calculated using the model [8] and summed with $k(M 1)=$ constant.

possible to recurrently and rather effectively determine $\rho$ and $k$ values, which accurately reproduce $I_{\gamma \gamma}$. Afterwards we may calculate and compare the experimental cascade population of any number of levels with the sufficiently determined assumptions about the ratios of the radiative strength functions of the primary and secondary gamma-transitions of one and the same energy and multipolarity. The complete reproduction of full set of experimental data is achieved by the repetition of the indicated above operations from 2 to, maximum, 3-4 times.

Examples of $\rho$ and $k$ determined from (1) for nuclei with different nucleon parity are presented in figures 1 and 2 . Analogous data for other nuclei are also presented in $[2,6]$. Inevitable systematical errors in determination of these parameters of nucleus and their potential dependence on the structure of an initial level of cascades make difficult the interpretation of observed $\rho$ and $k$ dependencies on energy of any selected nucleus. Most general tendencies in changing of $\rho$ and $k$ functions may be found by simple summing up of radiative strength functions and level density deviations from the interpolating exponential dependence $C \exp (b U)$ (for the region between known low lying levels and neutron resonances) in nuclei with different nucleon parity. Corresponding data are presented in figures 3-6. 

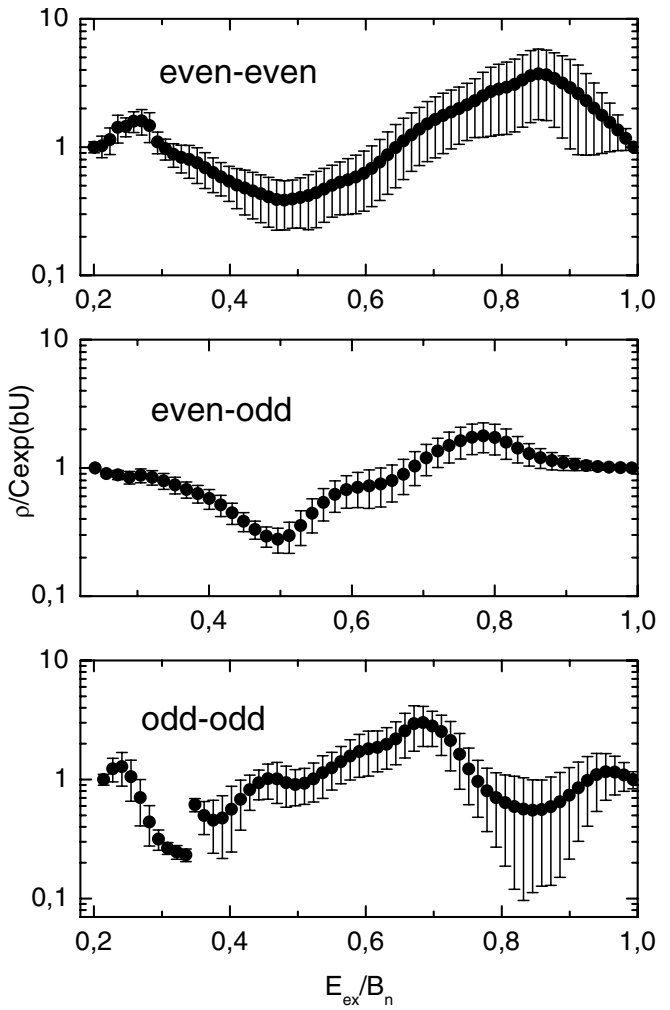

Fig. 3. Comparison of the mean values of level densities deviation for nuclei with different parities of their number of nucleons. The closed circles with error bars correspond the fit without using hypothesis [1].
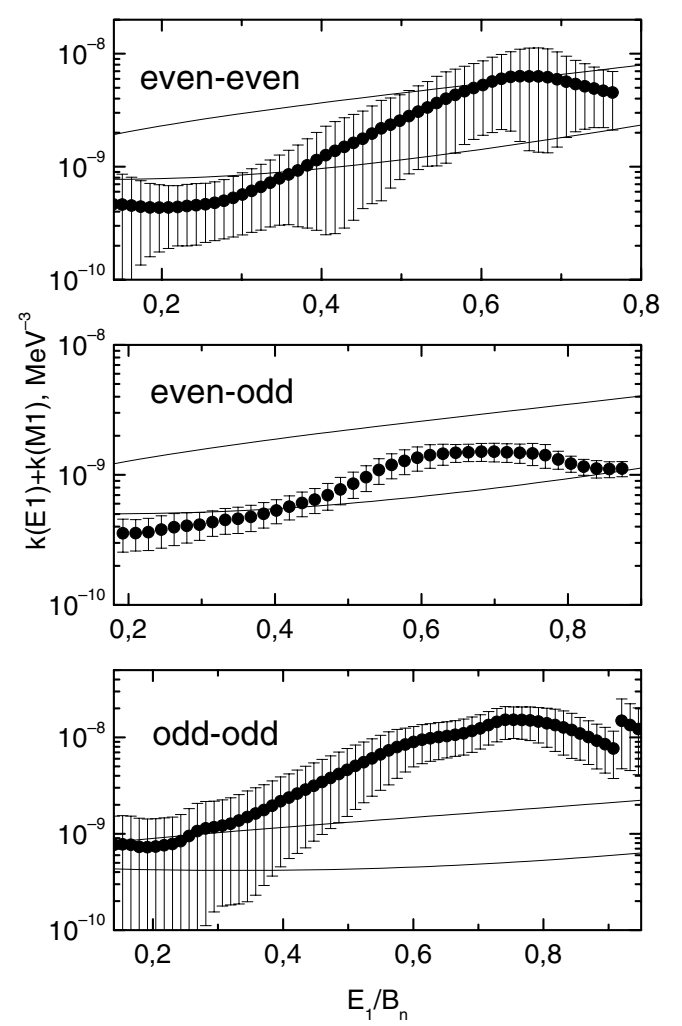

Fig. 4. Same as figure 3, for radiative strength functions.
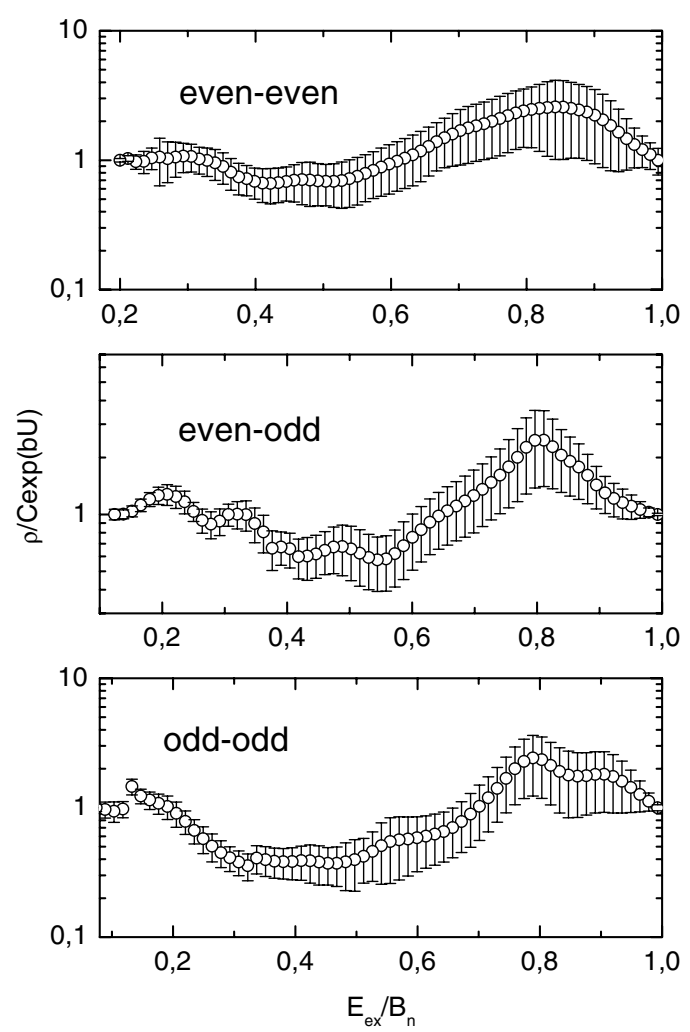

Fig. 5. Comparison of the mean values of level densities for nuclei with different parities of their number of nucleons. The open circles with error bars correspond the fit with using hypothesis [1].
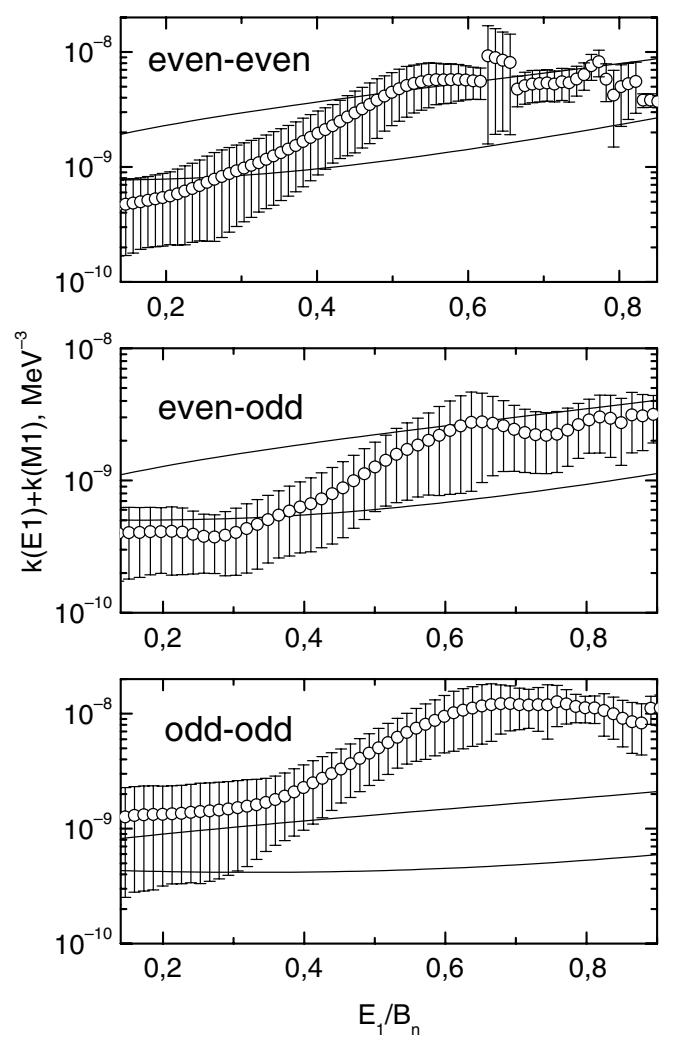

Fig. 6. Same as figure 5, for radiative strength functions. 


\section{Conclusion}

Experimental data on partial cross sections of two-step nuclear reactions make possible to minimize the influence of hypothesis [1] and partly consider [2] a real type of energy dependence of the interaction cross section of the reaction products with the residual nucleus. Therefore, determined from them, $\rho$ and $k$ values have the lowest systematical errors for the present. The comparison of data, obtained for a large number of nuclei from the intensities of two-step gammacascades without the use [2] of hypothesis [1] and with its use [6], indicates the high reliabilities of the results of studying the nuclear structure below $B_{n}$ with the quantitative analysis of these and, supposedly, analogous experiments on the charged particle beams.

Obvious and regular observation of changing of these parameters of nucleus at two excitation energies indicates sharp change of nucleus properties. Most probably it is caused [10] by breaking of two Cooper nucleon pairs at corresponding excitation energies of nucleus and change of their correlation function $\Delta$ from maximum value to zero in the $2 \Delta_{0}$ interval.

\section{References}

1. O. Bohr, B.R. Mottelson, Nuclear Structure, Vol. 1 (Benjamin, NY, Amsterdam, 1969).

2. A.M. Sukhovoj, V.A. Khitrov, Physics of Paricles and Nuclei 36(4), 359 (2005).

3. S.T. Boneva, V.A. Khitrov, A.M. Sukhovoj, Nucl. Phys. A 589, 293 (1995).

4. A.M. Sukhovoj, V.A. Khitrov, Physics of Atomic Nuclei 62(1), 19 (1999).

5. V.A. Khitrov, Li Chol, A.M. Sukhovoj, in Proceedings of XIth International Seminar Interaction of Neutrons with Nuclei, Dubna, May 2003, E3-2004-9 (Dubna, 2004), p. 98.

6. E.V. Vasilieva, A.M. Sukhovoj, V.A. Khitrov, Phys. At. Nucl. 64(2), 153 (2001).

7. W. Dilg, W. Schantl, H. Vonach, M. Uhl, Nucl. Phys. A 217, 269 (1973).

8. P. Axel, Phys. Rev. 126(2), 671 (1962).

9. S.G. Kadmenskij, V.P. Markushev, W.I. Furman, Sov. J. Nucl. Phys. 37, 165 (1983).

10. A.M. Sukhovoj, V.A. Khitrov, Physics of Particles and Nuclei 37(6), 899 (2006). 